\title{
Saccharide Primers Comprising Xylosyl-Serine Primed Phosphorylated Oligosaccharides Act as Intermediates in Glycosaminoglycan Biosynthesis
}

\author{
Yuya Otsuka ${ }^{\dagger, \ddagger}$ and Toshinori Sato*, ${ }^{*}$ \\ ${ }^{\dagger}$ Central Research Laboratories, Seikagaku Corporation, 1253, Tateno 3-chome, Higashiyamato-shi, Tokyo 207-0021, Japan \\ ${ }^{\ddagger}$ Department of Biosciences and Informatics, Faculty of Science and Technology, Keio University, 3-14-1 Hiyoshi, Kohokuku, \\ Yokohama, Kanagawa 223-8522, Japan
}

\section{Supporting Information}

\begin{abstract}
Xylosides have been used as an artificial initiator of glycosaminoglycan (GAG) biosynthesis to investigate its mechanism and to obtain these oligosaccharides. In GAG biosynthesis, phosphorylation on the xylose residue is a crucial step. However, little attention has been paid to phosphorylated oligosaccharides obtained from $\beta$-xylosides. In

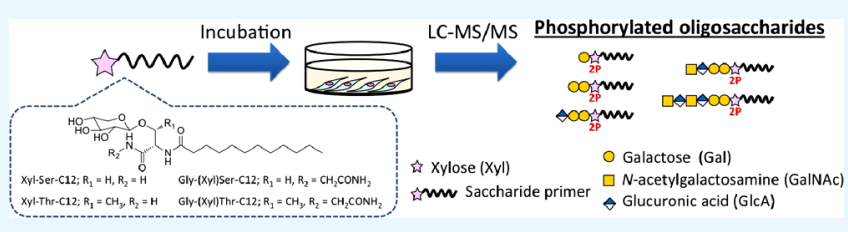
a previous study, we demonstrated that a novel $\beta$-xyloside, $N$-lauryl-O- $\beta$-xyloyranosyl-serinamide (Xyl-Ser-C12), had excellent GAG-type oligosaccharide priming ability, whereas phosphorylated oligosaccharides were not found in the primed oligosaccharides. This study examines the potential of Xyl-Ser-C12 and three of its derivatives for use as a probe to investigate the GAG biosynthesis mechanism. Glycosylated products were obtained by incubation of the $\beta$-xylosides in normal human dermal fibroblast cells and compared by liquid chromatography-electrospray ionization-mass spectrometry. By the optimized method to detect phosphorylated products, Xyl-Ser-C12 was demonstrated to prime not only GAG-type oligosaccharides but also a variety of xylose-phosphorylated products. Among the synthesized $\beta$-xylosides, those consisting of xylosyl-serine primed large amounts of phosphorylated and GAG-type oligosaccharides, whereas the others primed sialyloligosaccharides mainly. The majority of the phosphorylated products were considered to be GAG intermediates, which are less observed in nature. To our best knowledge, this is the first report showing that the amino acid residues around the Xyl attachment position strongly affect the phosphorylation efficiency and GAG chain-priming ability of $\beta$-xylosides. This study leads to the possibility of the use of $\beta$ xyloside as a probe to observe the Xyl phosphorylation process during GAG biosynthesis and investigate comparative glycosaminoglycomics between different cells.
\end{abstract}

\section{INTRODUCTION}

Glycosaminoglycans (GAGs), linear anionic polysaccharides, are mainly found in the extracellular matrix and play a key role in several biological events, such as cell-cell interaction, signal transduction, cancer progression, tumor migration, and anticoagulation. ${ }^{1}$ GAGs comprise alternating disaccharide units (hexuronic acid and hexosamine) and are classified by the disaccharide unit structure. Chondroitin sulfate (CS), dermatan sulfate (DS), and heparan sulfate (HS) are sulfated GAGs, which are sulfated in various positions, including the hydroxyl groups or amino groups. These GAGs exist as a side chain of proteoglycans (PGs) and are highly heterogeneous due to their chain lengths, sulfated degrees, and sulfated positions. This heterogeneity is attracting much interest because it makes it possible for GAGs to interact with various biological molecules, such as proteins. ${ }^{2}$

CS, DS, and HS are attached to a hydroxyl group on the serine residues in core proteins of $\mathrm{PGs}$ via a linkage tetrasaccharide (GlcA $\beta 1-3 \mathrm{Gal} \beta 1-3 \mathrm{Gal} \beta 1-4 \mathrm{Xyl} \beta 1-){ }^{3}$ Biosynthesis of the tetrasaccharide is initiated with xylosylation to the hydroxyl group on Ser in the core protein, followed by two galactosylations, after which the synthesis of the tetrasaccharide is accomplished by the addition of GlcA on the nonreducing end, Gal. ${ }^{4}$ The disaccharide units of CS, DS, and HS are elongated on the GlcA of the tetrasaccharide.

In GAG biosynthesis, phosphorylation on the Xyl is a key modification. ${ }^{3,5}$ The phosphorylation on the Xyl occurs transiently and is finally removed during construction of the linkage tetrasaccharide. It has recently been shown that this transient phosphorylation regulates the number and chain length of GAG elongated on the tetrasaccharide. ${ }^{6}$ However, it is difficult to observe the phosphorylated intermediate synthesized in cells because the phosphate group on the Xyl is mostly removed in mature GAG chains. ${ }^{7}$ The issue is even more complicated because the PGs in which tetrasaccharides are attached have structural varieties in their sequences. Thus, a simple and concise method to observe the linkage tetrasaccharides and their intermediates is needed for the investigation of the GAG biosynthesis mechanism.

Received: February 15, 2017

Accepted: May 22, 2017

Published: July 3, 2017 


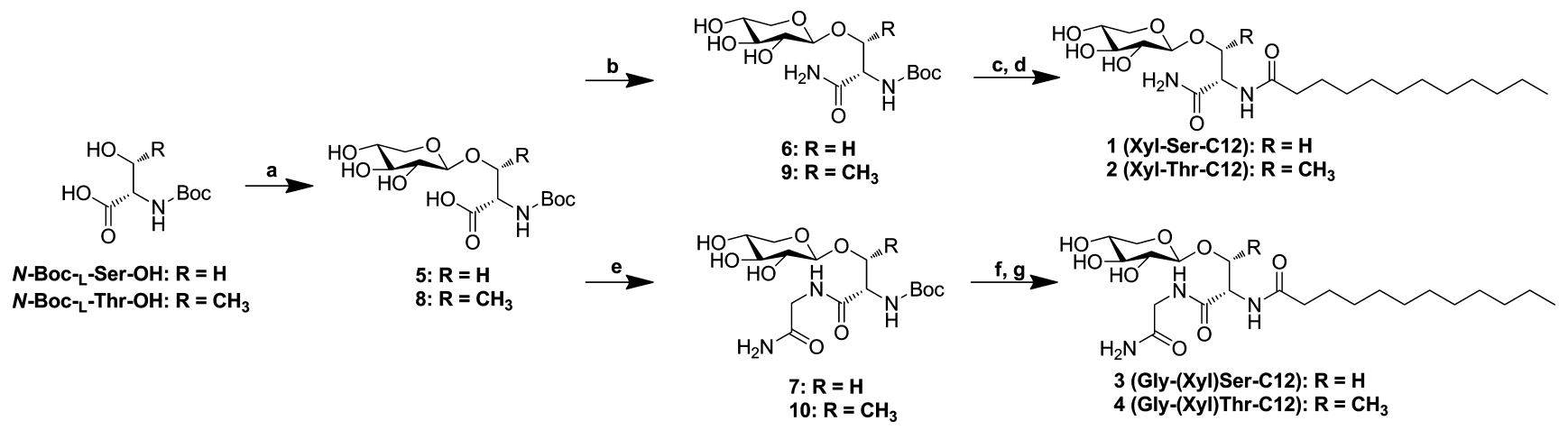

Figure 1. Chemoenzymatic synthesis of Xyl-Ser-C12 and its derivatives. Reagents and conditions: (a) xylo-oligosaccharides, $\beta 1-4$ xylanase, acetate buffer $\mathrm{pH}$ 5.0, $40{ }^{\circ} \mathrm{C}$; (b) ammonium salt $\left(\mathrm{HOBt}-\mathrm{NH}_{3}\right)$, 1-(3-dimethylaminopropyl)-3-ethylcarbodiimide hydrochloride (EDCI), acetonitrile $(\mathrm{MeCN})$, room temperature (r.t.); (c) $4 \mathrm{~mol} / \mathrm{L} \mathrm{HCl}$ in dioxane, r.t.; (d) 4-(4,6-dimethoxy-1,3,5-triazin-2-yl)-4-methylmorpholinium chloride (DMT-MM), $\mathrm{N}$-methylmorpholine (NMM), lauric acid, EtOH/ $\mathrm{H}_{2} \mathrm{O}(2 / 3)$, r.t.; (e) Gly- $\mathrm{NH}_{2}$, DMT-MM, NMM, MeCN, r.t.; (f) trifluoroacetyl, r.t.; (g) DMT-MM, NMM, lauric acid, $\mathrm{H}_{2} \mathrm{O}, 40{ }^{\circ} \mathrm{C}$.

$\beta$-Xylosides have been used to obtain GAG-type glycans due to their priming abilities of GAG oligosaccharides. For example, Okayama et al. first showed that $p$-nitrophenyl- $\beta$-xylopyranoside (Xyl-pNP) can prime GAG-type oligosaccharides when added to a chick cartilage cell culture. ${ }^{8}$ 4-Methylumbelliphenyl$\beta$-xylopyranoside, ${ }^{9} \beta$-estradiol-xylopyranoside, ${ }^{10}$ naphthol xylopyranosides, ${ }^{11-14} C$-xylopyranosides, ${ }^{15,16}$ and "click" xylopyranosides ${ }^{17-20}$ have also been applied for the elongation of GAG oligosaccharides. These $\beta$-xylosides have been used not only as a saccharide primer to obtain the GAG-type oligosaccharides but also as a chemical tool to explore species of glycans potentially produced by cells and colocation of glycosyltransferases on the endoplasmic reticulum and golgi apparatus. ${ }^{21}$ However, in most of these studies, the major focus was on the amount, molecular weight, and GAG types of primed oligosaccharides, whereas not much attention was paid to the intermediates of oligosaccharides primed on the $\beta$-xylosides.

In a previous study, it was demonstrated that $N$-lauryl- $O-\beta$ xyloyranosyl-serinamide (Xyl-Ser-C12), which was designed to resemble the attachment site of PG, showed excellent GAGtype oligosaccharide priming ability, including some intermediates of the linkage tetrasaccharides. ${ }^{22}$ The results imply that the amino acid residue in $\beta$-xylosides strongly affects their priming ability. Moreover, it is possible that a suitable $\beta$ xyloside that primes Xyl-phosphorylated oligosaccharides can be used as a chemical probe for the investigation of GAG biosynthesis. However, the relationship between the structure of the amino acids and the priming ability has not been examined, and phosphorylated oligosaccharides have not been found in the glycosylated products.

The aim of this study is to explore the potential of Xyl-Ser$\mathrm{C} 12$ and its derivatives as a chemical probe for investigating the GAG biosynthesis mechanism. $N$-Lauryl- $O-\beta$-xyloyranosylthreonamide (Xyl-Thr-C12) was chosen as a counterpart of $\mathrm{Xyl}$-Ser-C12 because threonine is another $\beta$-hydroxy amino acid, which is glycosylated by $\mathrm{O}$-glycans but not by GAG. $\mathrm{N}$ Lauryl-O- $\beta$-xyloyranosyl-serinyl-glycinamide (Gly-(Xyl)Ser$\mathrm{C} 12)$ and $N$-lauryl-O- $\beta$-xyloyranosyl-threonyl-glycinamide (Gly-(Xyl)Thr-C12) were chosen because of the effect of the Gly residue that is franked with the Ser residue in the PG consensus sequence. The four types of $\beta$-xylosides that have amino acid residues as aglycones were synthesized, and their priming ability was examined using the normal human dermal fibroblast (NHDF). The analytical procedure for priming oligosaccharides was optimized to detect phosphorylated products efficiently. The structure of the glycosylated products was deduced by tandem mass spectrometry (MS/MS) results and enzymatic digestion. The priming abilities of the $\beta$ xylosides were compared, and the relationship between the structure of the aglycones and the priming ability is discussed.

\section{RESULTS AND DISCUSSION}

Synthesis of Xyl-Ser-C12 (1) and Its Derivatives (2-4). The synthesis scheme of Xyl-Ser-C12 (1) and its derivatives is shown in Figure 1. In a previous study, Xyl-Ser-C12 was chemically synthesized from 2,3,4-tri-O-acetyl-D-xylopyranosyltrichloroacetimidate as a glycosyl donor. However, this chemical synthesis required multiple steps to control both the reaction sites and anomeric selectivity of xylose, necessitating large amounts of reagents and organic solvent. To avoid this problem, we chose chemoenzymatic condensation using a glycosidase to synthesize $\beta$-xylosylated amino acids. In chemoenzymatic condensation, high selectivity of reaction sites and anomeric configuration can be achieved because of the stereoselectivity of glycosidases used for synthesis. ${ }^{23-32}$ In this study, we chose xylo-oligosaccharides as the glycosyl donors, $\mathrm{N}$ tert-butoxycarbonyl-serine (Boc-Ser-OH) or N-Boc-threonine (Boc-Thr-OH) as acceptors, and commercially available $\beta 1-4$ xylanase from Trichoderma longibrachiatum. The chemoenzymatic condensations were carried out at $40{ }^{\circ} \mathrm{C}, \mathrm{pH} 5.0$, for 4 days to obtain monoxylosylated amino acids ( 5 and 8 ). In ${ }^{1} \mathrm{H}$ NMR spectra of the monoxylosylated amino acids, the peaks of the anomeric protons appeared at around $4.4 \mathrm{ppm}$, and the coupling constants were $8 \mathrm{~Hz}$ (Figures S1 and S2), confirming that the purified products had the $\beta$-anomer configuration. The yields of the chemoenzymatic condensations were $11.1 \%$ for $\mathrm{N}$-Boc- $\mathrm{O}$ - $(\beta$-xylopyranosyl)-L-serine $(5)$ or $12.5 \%$ for $\mathrm{N}$-Boc- $\mathrm{O}$ - $(\beta$-xylopyranosyl $)$-L-threonine $(8)$. These results were reasonable, and the chemoenzymatic reactions using $\beta 1-4$ xylanase were properly proceeded because Nilsson et al. reported that yields of Boc-Ser glycosides were $4-14 \%$ and Boc-Thr glycosides were $4-8 \%$ using $\beta$-mannosidase and $\beta$-galactosidase as catalysts. ${ }^{32}$

Conversion of 5 and 8 into the final products $(1-4)$ was carried out in accordance with the $\mathrm{N}$-Boc chemistry for peptide synthesis. For conversion of carboxyl groups into amides, the reactions were carried out with 1-hydroxy- $1 H$-benzotriazole, HOBt- $\mathrm{NH}_{3}$, and EDCI. ${ }^{33}$ Condensation of $\mathbf{5}$ or $\mathbf{8}$ with 

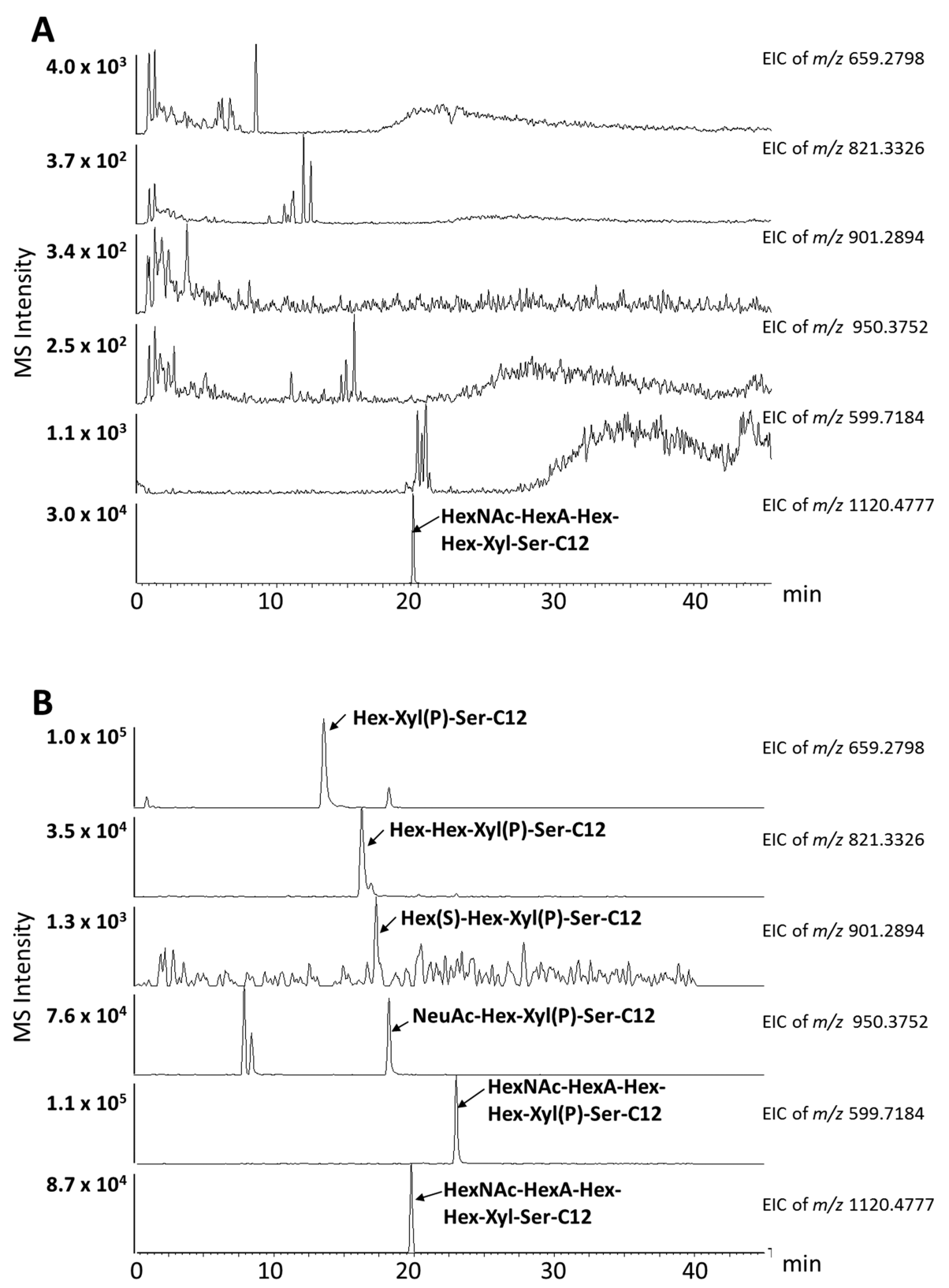

Figure 2. Flushing with phosphoric acid before the analysis can improve the peak shapes of the phosphorylated products. (A) EICs of the glycosylated products before phosphorylated acid wash. (B) EICs of the phosphorylated products after phosphoric acid wash.

glycinamide was carried out using DMT-MM. Condensation with lauric acid was carried out in a two-step synthesis involving removal of the $N$-Boc group with acid and condensation using DMT-MM. The overall yields of the final products were $4.10 \%$ for Xyl-Ser-C12, $4.91 \%$ for Xyl-Thr-C12, $5.66 \%$ for Gly(Xyl)Ser-C12, and 5.06\% for Gly-(Xyl)Thr-C12. Compared to the results of the previous report, the number of synthesis steps was reduced from 10 to 3 .

The Xyl-Ser-C12 synthesized in this study showed negative specific rotation. This was inconsistent with the previous report. $^{22}$ In the report, deacetylation was carried out with sodium methoxide, and this strong base treatment might have caused racemization of the L-serine residue. Taking these facts into consideration, Xyl-Ser-C12 synthesized in the previous study might have been racemized. In this study, Xyl-Ser-C12 and other $\beta$-xylosides showed negative specific rotation; hence, the racemization did not occur during the syntheses of these $\beta$ - xylosides. In addition, some of the literature has reported that D-xylosyl-L-serine and its derivatives have negative specific rotation. ${ }^{34,35}$ Consequently, we consider that the Xyl-Ser-C12 synthesized in this study has a D-xylosyl-L-serine structure, and the synthetic scheme using chemoenzymatic condensation is less laborious and more stereoselective.

Method Optimization and Structure Analysis of Phosphorylated Products. As mentioned in the Introduction section, phosphorylation on the Xyl residue in the linkage tetrasaccharides is an important step in GAG biosynthesis. However, phosphorylated GAGs are rarely found in nature, and only phosphorylated products of $\beta$-xylosides have been reported. ${ }^{36,37}$ The reasons given for this include poor recovery of the phosphorylated products from the cell culture, $4,38-40$ incomparability of the analytical apparatus and conditions for the phosphorylated products, and difficulty in distinguishing the phosphorylated and sulfated products. Hence, in this study, 
we optimized the protocol for extraction, liquid chromatography-mass spectrometry (LC-MS) conditions, and applied high-resolution mass spectrometry.

In the LC-MS analysis, it is well-known that phosphorylated compounds are easily absorbed on the titanium and the stainless steel used in the LC system, and this absorption causes poor peak shapes of the phosphorylated compounds. ${ }^{41}$ When the glycosylated products were analyzed under conventional conditions, the phosphorylated products gave extremely broad peaks or were not detected at all (Figure 2A). To avoid this absorption, we chose to flush the $\mathrm{LC}$ system with $\mathrm{H}_{3} \mathrm{PO}_{4}$ solution before every analysis. By flushing the LC-MS system with $20 \mathrm{mM} \mathrm{H}_{3} \mathrm{PO}_{4}$ in $50 \% \mathrm{MeCN}$ before every analysis, the peak shapes of the phosphorylated products were improved dramatically without changing the retention time, peak shape, or sensitivity to other glycosylated products (Figure 2B).

The glycosylated products obtained from the NHDF cell culture were prepared and analyzed by the optimized method. Table 1 shows the structure, $\mathrm{m} / z$ value, ion type, retention time, proportions, and fragment ions of the MS/MS spectrum of the detected glycosylated product. The structures of the glycosylated products were deduced from the MS/MS spectra and the results of enzymatic digestion. In particular, phosphorylated and sulfated products were determined by accurate $m / z$ values and observation of $m / z 78.9585\left(\left[\mathrm{PO}_{3}\right]^{-}\right)$ in the MS/MS spectra. Twenty-nine glycosylated products were detected from $1.5 \mathrm{~mL}$ of the NHDF cell culture, of which 11 were considered to be phosphorylated products.

Figure 3 shows the structural analysis of the disaccharide $(\mathrm{m} /$ $z$ 659.2798) as an example. In the MS/MS spectrum (Figure $3 \mathrm{~A}),\left[\mathrm{PO}_{3}\right]^{-}(\mathrm{m} / z 78.9601)$ was clearly observed and hence the disaccharide was considered to be phosphorylated. ${ }^{42}$ In addition, $m / z 497.2288\left(\mathrm{Y}_{1}\right), 391.0667\left(\mathrm{C}_{2}\right)$, and 373.0561 $\left(\mathrm{B}_{1}\right)$ meant glycoside bond cleavage ions, suggesting that the phosphorylation occurred on the Xyl. However, in the phosphorylated product, peaks at $\mathrm{m} / \mathrm{z} 241.0112$ and 259.0222 were also clearly observed (shown by arrows in Figure 3A). These ions implied the existence of a phosphorylated hexose residue in the products, a phenomenon inconsistent with the existence of the phosphorylated $\mathrm{Xyl}$ residue. To clarify this point, the glycosylated products were digested by $\beta$-galactosidase, followed by the LC-MS/MS analysis. Figure $3 \mathrm{~B}$ shows the extracted ion chromatograms (EICs) of $m / z \quad 659.2798$ and 497.2270 (Xyl(P)-Ser-C12) before and after $\beta$-galactosidase digestion, respectively. The peak height at $11.5 \mathrm{~min}$ on the EIC of $\mathrm{m} / z 659.2798$ (Hex$\mathrm{Xyl}(\mathrm{P})$-Ser-C12) decreased considerably after digestion, whereas that at $5.7 \mathrm{~min}$ on the EIC of $\mathrm{m} / z 497.2270$ (Xyl(P)-Ser$\mathrm{C} 12)$ largely increased after digestion. On the basis of these results, the major component of the phosphorylated disaccharide $(m / z 659.2798)$ was deduced to be $\mathrm{Gal} \beta$-Xyl(P)Ser-C12. The fragment ions $m / z 241.0112$ and 259.0222 in the MS/MS may have been detected due to migration of the phosphate group during MS/MS excitation ${ }^{43}$ or coelution of $\operatorname{Gal}(\mathrm{P}) \beta$-Xyl-Ser-C12.

As in the case of the phosphorylated disaccharide, other glycosylated products that gave $\left[\mathrm{PO}_{3}\right]^{-}$ions in their MS/MS spectra were considered to be phosphorylated products. These phosphorylated products were deduced to be phosphorylated on the Xyl residue because $\mathrm{Y}_{1}(\mathrm{~m} / \mathrm{z} 497.23)$ or $\mathrm{Y}_{2}(\mathrm{~m} / z$ 659.28) ions were observed in their MS/MS spectra (Table 1 ). Thus, the phosphorylated products were concluded to be intermediates of the linkage tetrasaccharide and GAG oligosaccharides. The major reason why the phosphorylated products were not detected in previous studies is probably due to the absorption of these products. This result demonstrates the feasibility of Xyl-Ser-C12 for use as a chemical probe to investigate the GAG biosynthesis mechanism.

Interestingly, not only Xyl-phosphorylated di-, tri-, and tetraoligosaccharides but longer phosphorylated pentasaccharides $(\mathrm{m} / z 599.7184$ and 701.2581$)$ and a heptasaccharide $(\mathrm{m} / z$ $789.2741)$ were also detected. The phosphorylated pentasaccharide $(\mathrm{m} / z$ 599.7184) could be partly digested by heparitinases (Figure 4A); the major structure of the phosphorylated pentasaccharide was deduced to be GlcNAc $\alpha 1-4$ HexA-Hex-Hex-Xyl(P)-Ser-C12. In contrast, the phosphorylated heptasaccharide $(\mathrm{m} / z$ 789.2741) could be digested by chondroitinase $\mathrm{ABC}$ (C-ABC) and chondroitinase ACII (CACII) but was not digested by heparitinases (Figure 4B). Therefore, the structure of the heptasaccharide would be GalNAc $\beta 1$-4GlcA $\beta 1$-3GalNAc $\beta 1$-4GlcA-Hex-Hex-Xyl(P)-Ser$\mathrm{C} 12$. Izumikawa et al. demonstrated the phosphorylated linkage oligosaccharides to be an intermediate of the immature GAG chain resulting from an imbalance of GAG xylosylkinase-named family with sequence similarity 20, member B (FAM20B), xylose phosphatase, and chondroitin $\mathrm{N}$-acetylgalactosaminyltransferase-1. ${ }^{6}$ Their results indicate that the phosphorylation on $\mathrm{Xyl}$ residues may be a discrimination tag to sort the linkage oligosaccharides into GAG chain elongation or termination and that these enzymes work in harmony to regulate the number, length, and amount of GAG chains elongated on PGs. In this study, both phosphorylated and nonphosphorylated oligosaccharides that have a linkage tetrasaccharide structure were detected. Thus, the ratio of phosphorylated and nonphosphorylated oligosaccharides probably represents the capability of GAG biosynthesis of the examined cells.

Structural Analysis of the Glycosylated Products by GAG Lyase Digestion. To determine the GAG types of the elongated oligosaccharides, the glycosylated products were digested by GAG lyases, followed by the LC-MS/MS analysis. Figure 5 shows the structural analysis of heptasaccharides $(\mathrm{m} / z$ 749.2910). In the chromatograms (Figure 5A), the untreated sample gave a minor peak at $24.49 \mathrm{~min}$ and a major peak at $25.97 \mathrm{~min}$. After digestion with $\mathrm{C}-\mathrm{ABC} / \mathrm{C}-\mathrm{ACII}$, the major peak completely disappeared, whereas the minor peak remained intact. In contrast, the minor peak completely disappeared by heparitinase digestion, whereas the entire major peak remained. In addition, the cross-ring cleavage ion, ${ }^{2,5} \mathrm{~A}_{3}(\mathrm{~m} / z$ 480.1401), was observed in the MS/MS spectra of $\mathrm{m} / z 749.2910$ at the minor peak (Figure 5B), indicating the existence of the -HexA1-4HexNAc- structure in the sequence. The cross-ring cleavage ion was not observed in the spectra at the major peak (Figure 5C). Other oligosaccharides composed of repeating disaccharide units were digested by $\mathrm{C}-\mathrm{ABC} / \mathrm{C}-\mathrm{ACII}$ but not by heparitinase (Figures 4B and S3). Therefore, the heptasaccharide at the minor peak was considered to be an HS-type oligosaccharide and that at the major peak a CS-type oligosaccharide.

Structural Analysis of Sialyloligosaccharides. Besides GAG-type oligosaccharides, many sialyloligosaccharides are found in the glycosylated products. Among these sialyloligosaccharides, $\alpha 2-3$ sialosides are considered to be an unnatural type of oligosaccharides, resulting from misrecognition of $\beta$ xylosides by the glycolipid biosynthesis pathway ${ }^{21}$ or an endcapping structure instead of phosphorylated $\mathrm{Xyl} .{ }^{6}$ To confirm the structure of the sialoside bond of the glycosylated products, 


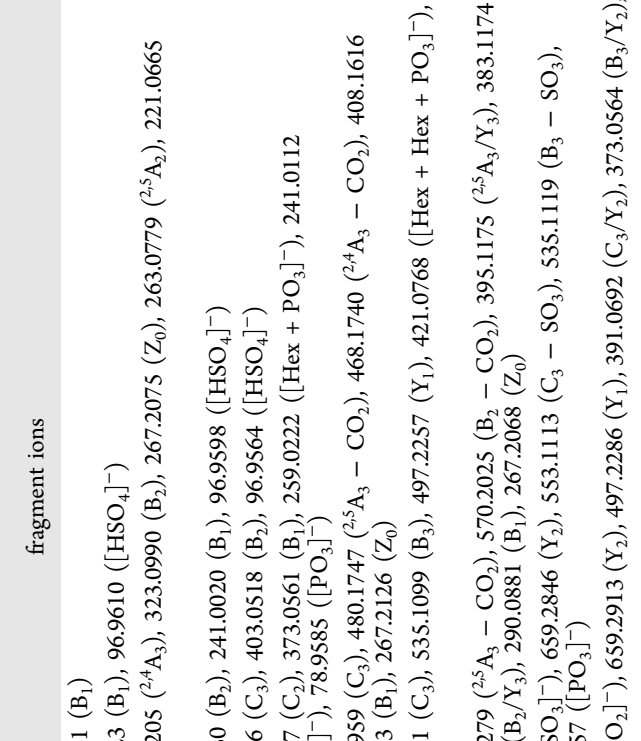

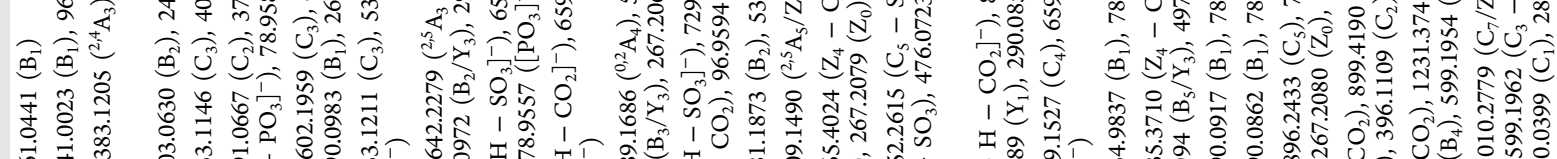

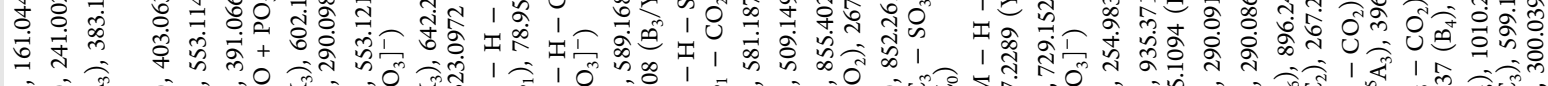

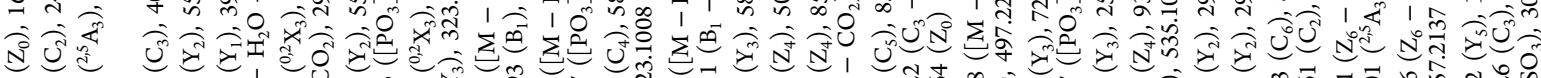

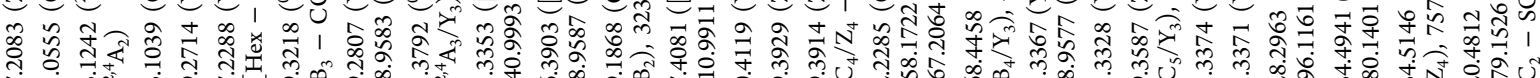

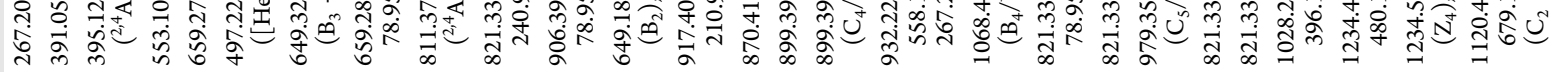

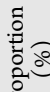
ఠ

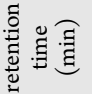

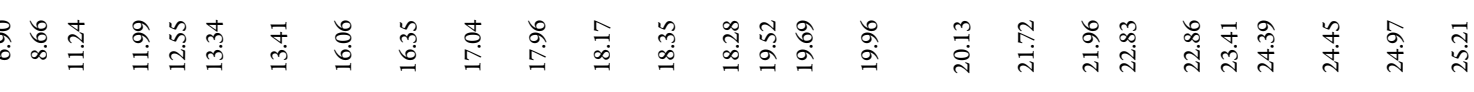

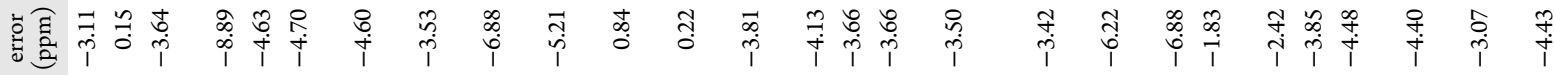

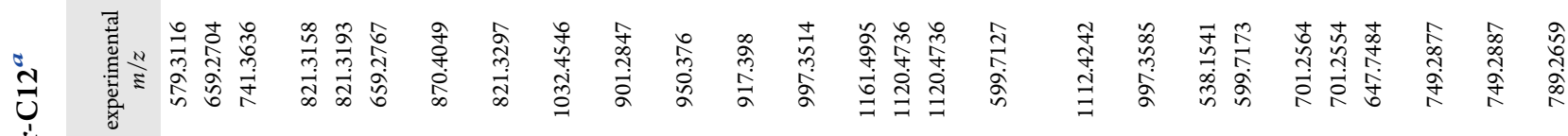

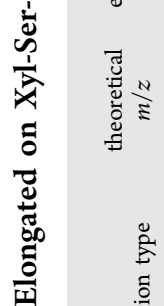

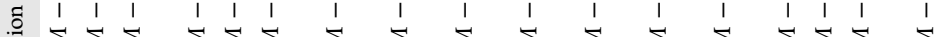

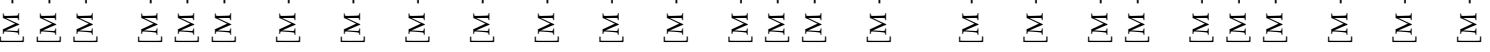

$+\infty$

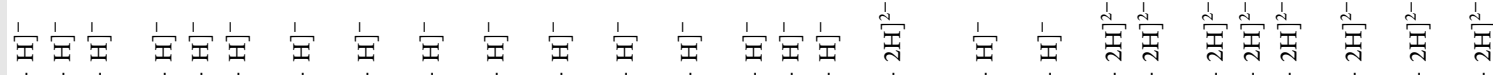


they were digested by $\alpha 2-3$ sialidase or $\alpha 2-3,6$ sialidase and then analyzed by LC-MS/MS. The result is shown in Figure 6. NeuAc-Hex-Xyl-Ser-C12 $(\mathrm{m} / z$ 870.4089), the most abundant sialyloligosaccharide, completely disappeared by digestion with both sialidases (Figure 6A). Similarly, NeuAc-Hex-Xyl(P)-Ser$\mathrm{C} 12(\mathrm{~m} / z$ 950.3752) and NeuAc-Hex-Hex-Xyl-Ser-C12 $(\mathrm{m} / z$ 1032.4617) were digested by both sialidases (Figure 6B,C), so the sialoside bonds of these products were determined to be an $\alpha 2-3$ sialoside bond. In contrast, NeuAc-Hex-Hex-Xyl(P)-Ser$\mathrm{C} 12(\mathrm{~m} / z 1112.4280)$ was digested only by $\alpha 2-3,6$ sialidase and not by $\alpha 2,3$ sialidase (Figure 6D). This result suggests the structure to be an $\alpha 2-6$ sialoside.

NeuAc $\alpha 2-3 \mathrm{Hex}-\mathrm{Xyl}$ is regarded as an end-capping structure when disaccharide structure $\mathrm{Hex}-\mathrm{Xyl}$ is not phosphorylated properly by FAM20B. ${ }^{5}$ Therefore, the proportions of this sialyloligosaccharide show a tendency of Xyl-Ser-C12 to not be incorporated into the GAG biosynthetic pathway. Moreover, NeuAc $\alpha 2-3$ Hex-Hex-Xyl is also considered to be an endcapping structure because the $\mathrm{C} 3$ position of the nonreducing end, Hex, where GlcA should be attached, was occupied by sialic acid. In contrast, some sialyloligosaccharides that were phosphorylated on the Xyl residue were also detected. This result did not agree with the GAG biosynthesis mechanism controlled by FAM20B, suggesting that the sialylation might occur by a different biosynthesis mechanism.

In addition, the sialyltetrasaccharide (NeuAc $\alpha 2-6 \mathrm{Hex}-\mathrm{Hex}-$ Xyl(P)-Ser-C12) had another sialoside bond structure. Recently, sialylation on the Gal residue of the linkage tetrasaccharide in the human inter- $\alpha$-trypsin inhibitor was reported. ${ }^{44}$ This implies that $\alpha 2-6$ sialoside might be added on the Gal residues of the linkage tetrasaccharide during GAG biosynthesis. Therefore, NeuAc $\alpha 2-6-H e x-H e x-X y l(P)-S e r-C 12$ might be a novel intermediate structure of GAG biosynthesis. On the basis of these results, sialylation on the $\beta$-xyloside might be a key factor to understanding the GAG biosynthetic mechanism. In this regard, Xyl-Ser-C12 could be a powerful tool to investigate the GAG biosynthetic mechanism because $\mathrm{Xyl}-\mathrm{Ser}-\mathrm{C} 12$ is able to prime phosphorylated, GAG-type, and sialyloligosaccharides all at the same time.

Quantitative Analysis of the Glycosylated Products Obtained from $\boldsymbol{\beta}$-Xylosides. To confirm the relationship between the structure and priming ability of each $\beta$-xyloside, the proportions of the glycosylated products were calculated and compared. In the same manner as that for the glycosylated products of Xyl-Ser-C12, those of other $\beta$-xylosides were deduced by LC-MS/MS and enzymatic digestion (shown in Supporting Information). The proportions were calculated from the peak areas of the glycosylated products. The results are shown in Table S4 and Figure 7. Among the $\beta$-xylosides, Xyl-Ser-C12 primed the maximum amount of glycosylated products (Table S4). Among the glycosylated products of XylSer-C12, the most abundant product was a phosphorylated pentasaccharide (HexNAc-HexA-Hex-Hex-Xyl(P)-Ser-C12) (20.91\%). The sum of the proportion of phosphorylated products obtained from Xyl-Ser-C12 is about 50\%. These phosphorylated products have not been detected in previous studies $^{22,45}$ (Figure 7A). These results suggest that Xyl-Ser-C12 works as an appropriate substrate for Xyl phosphorylation, and the phosphorylated products were easily excreted into the medium. Furthermore, the sum of the proportion of GAG-type oligosaccharides, which were composed of the linkage tetrasaccharides, was about $40 \%$ (Figure 7B). Taking into consideration the fact that the phosphorylated products were 


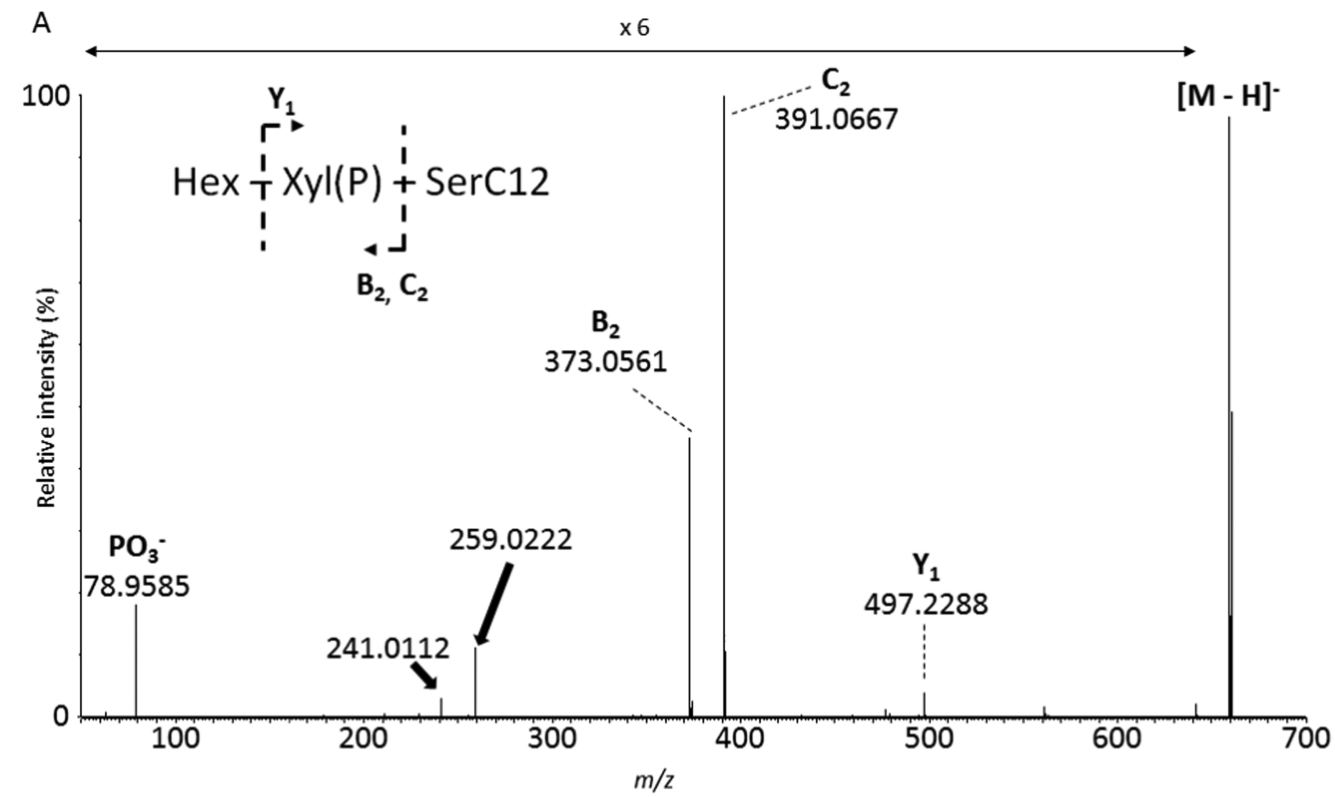

B

Hex-Xyl(P)-Ser-C12
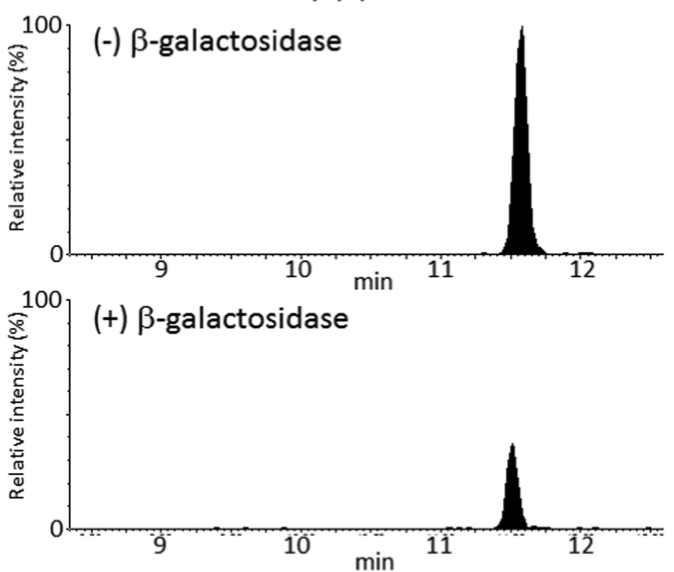

Xyl(P)-Ser-C12
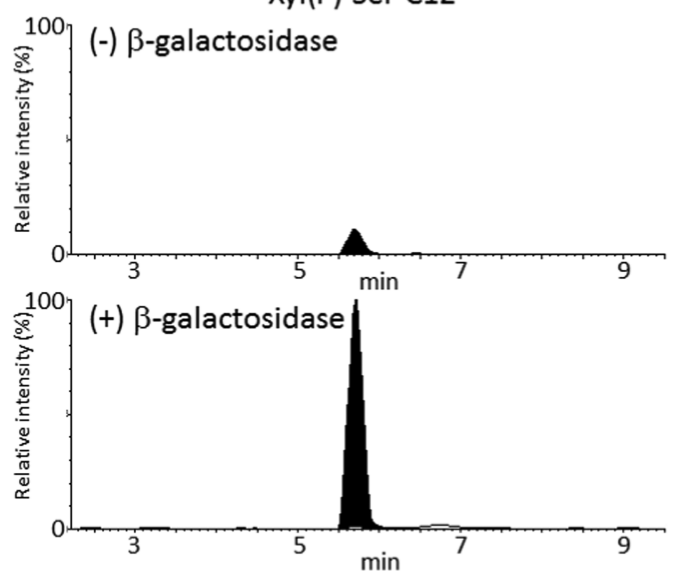

Figure 3. Structure analysis of the phosphorylated disaccharide $(\mathrm{m} / z$; 659.2798). (A) MS/MS spectrum of $m / z 659.2798$. Assignment of the fragment ions is described in the figure. Arrows indicate $\mathrm{m} / z 241.0112$ and 259.0222 . (B) Comparison of EIC profiles before and after $\beta$ galactosidase digestion. The ranges of the vertical axis are set equal.

the intermediates of GAG biosynthesis, a large part of Xyl-Ser$\mathrm{C} 12$ was incorporated into the GAG biosynthesis pathway. The total proportion of the sialyloligosaccharides was approximately $17 \%$. Therefore, some part of Xyl-Ser-C12 taken by NHDF cells may be recognized by sialyltransferases.

Similar to Xyl-Ser-C12, Gly-(Xyl)Ser-C12 also initiated mainly GAG-type oligosaccharides. The most abundant product was the phosphorylated pentasaccharide (HexNAcHexA-Hex-Hex-Xyl(P)-GlySer-C12) (36.75\%). In comparison with Xyl-Ser-C12, phosphorylated products and GAG-type oligosaccharides were slightly but not significantly increased (Figure 7A,B). In addition, it is notable that the amount of sialyloligosaccharides elongated on Gly-(Xyl)Ser-C12 was significantly lower than that on Xyl-Ser-C12 (Figure 7C). These results suggest that the Gly residue flanked by the Ser residue in Gly-(Xyl)Ser-C12 affects the efficiency of $\mathrm{Xyl}$ phosphorylation and sialylation. Gly-(Xyl)Ser-C12 would be a better primer to construct GAG-like oligosaccharide libraries because $\alpha 2-3$ sialylated oligosaccharides, which are the end- capping structure, were undesirable to obtain phosphorylated and GAG-type oligosaccharides.

Xyl-Thr-C12 and Gly-(Xyl)Ser-C12 gave fewer species of elongated oligosaccharides than either Xyl-Ser-C12 or Gly(Xyl)Ser-C12 (Table S4). They initiated large amounts of sialyloligosaccharides, such as NeuAc $\alpha 2-3 \mathrm{Hex}-\mathrm{Xyl}-\mathrm{R}$, and small amounts of both phosphorylated and GAG-type oligosaccharides (Figure $7 \mathrm{~A}-\mathrm{C}$ ). Therefore, these $\beta$-xylosides, which are composed of a Xyl-Thr residue, are unlikely to work well for Xyl phosphorylation.

\section{CONCLUSIONS}

In this study, we established an analytical method for phosphorylated oligosaccharides elongated on the saccharide primers, by which a wide variety of oligosaccharides could be observed using LC-MS/MS. Most of these oligosaccharides seemed to be synthesized in accordance with the GAG biosynthetic pathway (Figure 8). These results indicate that the $\beta$-xylosides prime phosphorylated GAG-type oligosaccharides in living cells. In addition, the comparison of the relative 
A

HexNAc-HexA-Hex-Hex-XyI(P)-Ser-C12
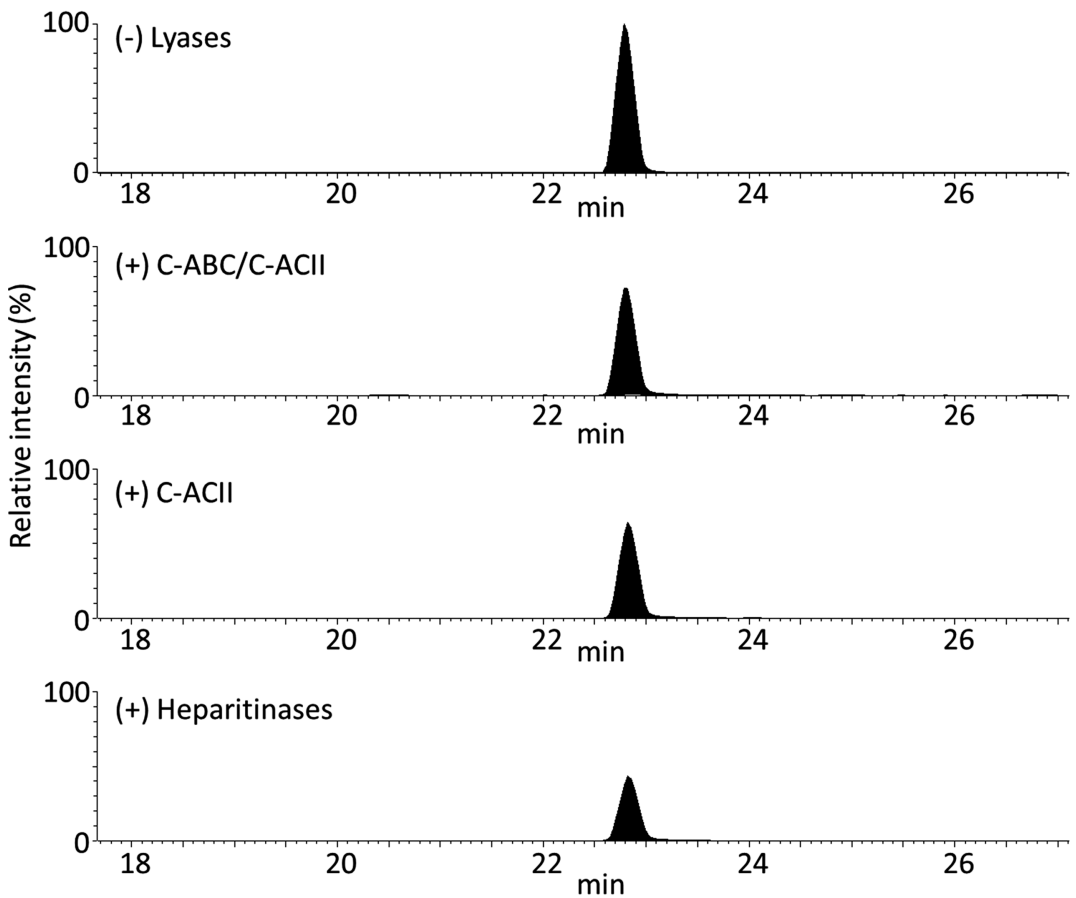

B

(HexNAc-HexA) ${ }_{2}$-Hex-Hex-Xyl(P)-Ser-C12
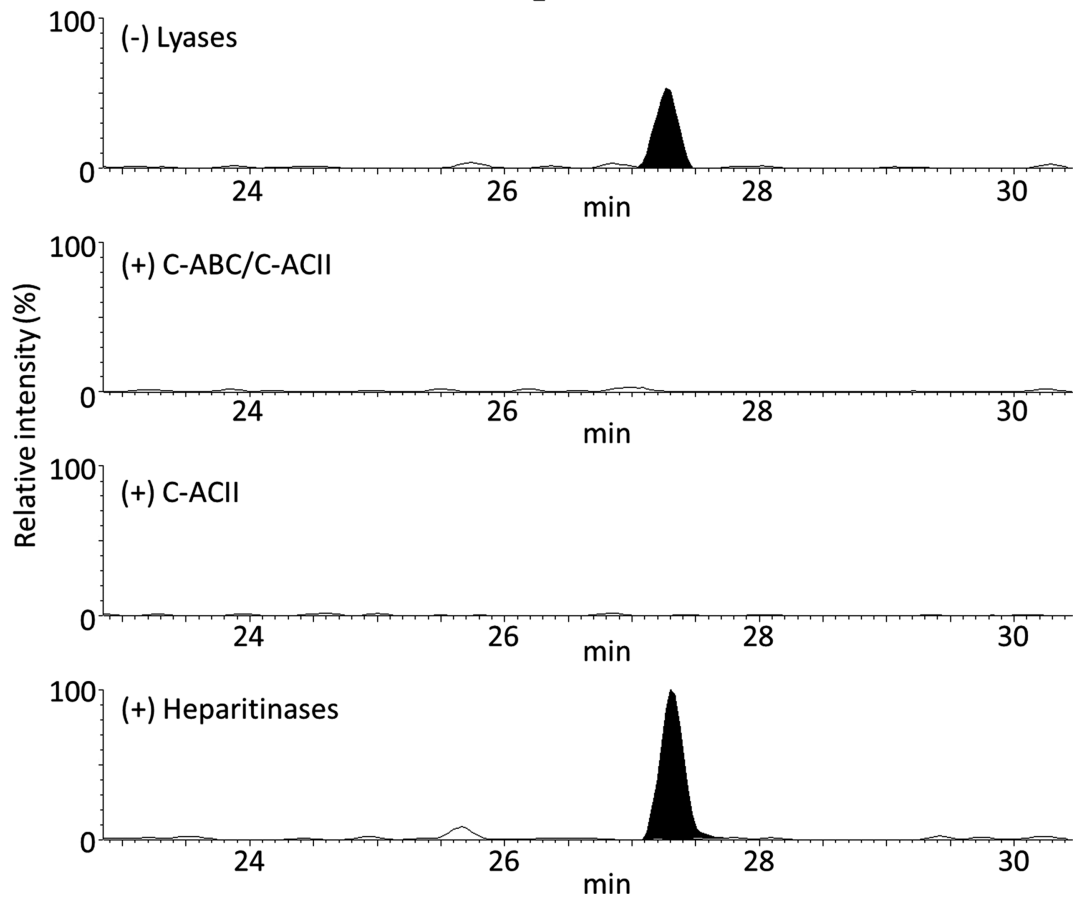

Figure 4. Comparison of EIC profiles before and after GAG lyase digestion. (A) EIC profiles of phosphorylated pentasaccharide (HexNAc-HexAHex-Hex-Xyl(P)-Ser-C12; $m / z$ 599.7184). (B) EIC profiles of phosphorylated heptasaccharide (HexNAc-HexA-HexNAc-HexA-Hex-Hex-Xyl(P)Ser-C12; $m / z$ 789.2741). The ranges of the vertical axis are set equal.

amounts of these oligosaccharides revealed that the proportions of phosphorylated oligosaccharides and sialylated oligosaccharides were completely different between the primers. To our best knowledge, this is the first report to demonstrate that the amino acid residues around the $\mathrm{Xyl}$ attachment position strongly affect phosphorylation efficiency. Taking into consideration the GAG biosynthetic pathway, our results suggest that both phosphorylation and $\alpha 2-3$ sialylation are related to the GAG priming ability of the primers. Notably, these oligosaccharides included phosphorylated $\alpha 2-6$ sialyloligosaccharides, implying that both sialylation and Xyl phosphorylation might orchestrate the GAG biosynthesis mechanism. However, the function of the $\alpha 2-6$ sialylation in the GAG biosynthesis mechanism remains to be discussed further, thus 
A
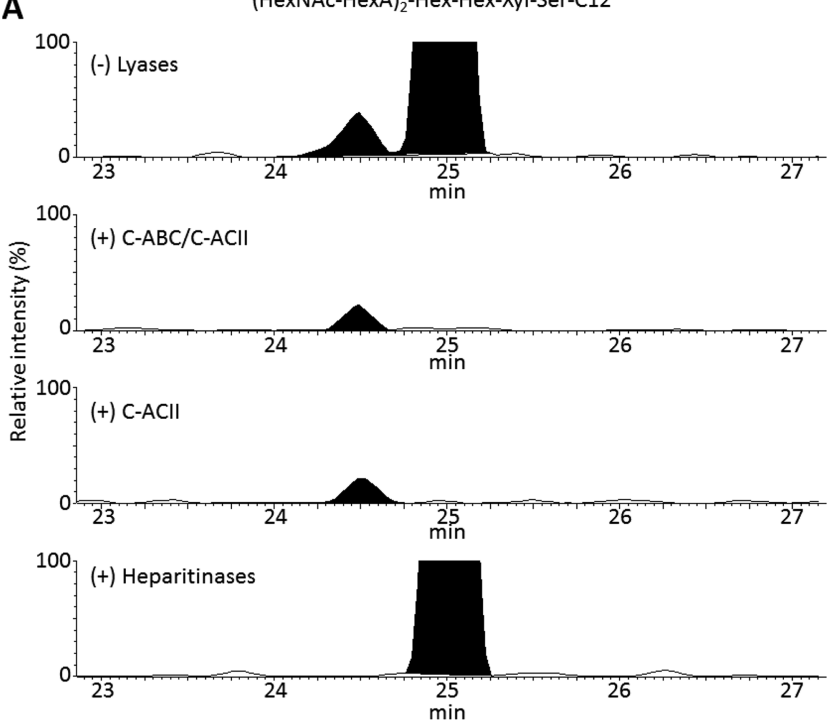

B

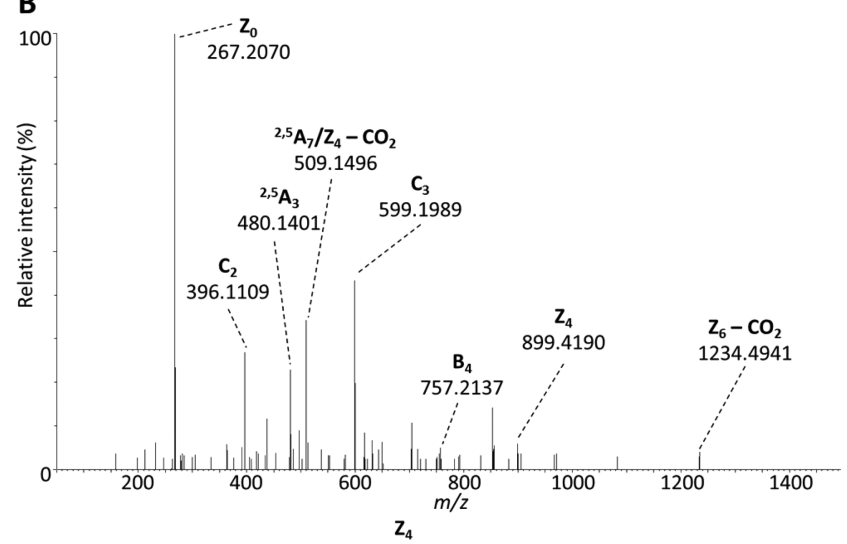

C

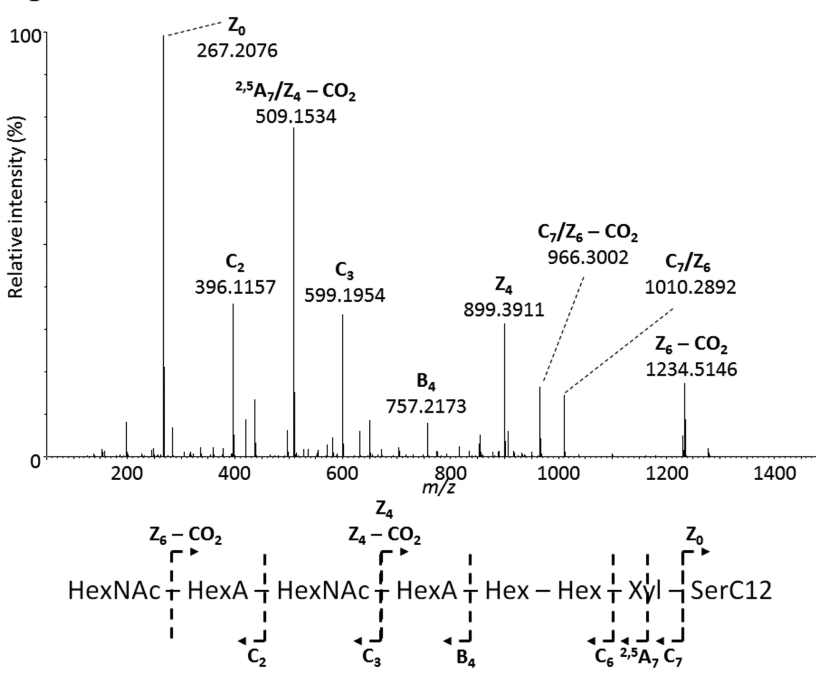

Figure 5. Structural analysis of the heptasaccharides $(m / z 749.2910)$. (A) EIC profiles of the heptasaccharides. The ranges of the vertical axis are set equal. (B) The MS/MS spectrum of the heptasaccharide at $24.4 \mathrm{~min}$. (C) MS/MS spectrum of the heptasaccharide at $24.9 \mathrm{~min}$.

in-depth analysis of the phosphorylated sialyloligosaccharides is needed.

$\beta$-Xylosides with amino acid residues in their aglycone were synthesized by chemoenzymatic synthesis to examine the effect of the aglycone structure on the priming ability. The amino acid residues strongly affect the structure of oligosaccharides, especially phosphorylated oligosaccharides. The $\beta$-xylosides synthesized in this study demonstrated high priming ability for a wide variety of oligosaccharides, providing a chemical tool not only to obtain GAG-type oligosaccharides but also to investigate the GAG biosynthesis mechanism. This study is an important step in the development of a new methodology using $\beta$-xylosides to clarify the GAG biosynthesis mechanism and for the comparison of glycosaminoglycomics between different cells.

\section{METHODS}

Materials. NHDF cells, NHDF growth supplements, gentamicin, amphotericin B, and FibroLife S2 Medium Complete Kit were purchased from Kurabou, Japan. Medium 106 was purchased from Thermofisher Scientific, Japan. All other reagents were purchased from Sigma-Aldrich, Japan, Wako Pure Chemical Industries, and Tokyo Chemical Industries. Unless otherwise stated, all commercially available reagents and solvents were of reagent grade and used without purification.

Synthesis of $\beta$-Xylosides (Compounds 1-4). All data and procedures for the synthesis of $\beta$-xylosides are described in the Supporting Information.

GAG Priming in Cells. To investigate the priming ability of the $\beta$-xylosides, glycosylation of the $\beta$-xylosides was examined with NHDF cells. NHDF cells were cultured in medium 106 with NHDF growth supplements, gentamicin, and amphotericin $\mathrm{B}$ at $37^{\circ} \mathrm{C}$ in a humidified atmosphere containing $5 \% \mathrm{CO}_{2}$. NHDF cells $\left(4 \times 10^{5}\right)$ in a six-well plate were seeded with the FibroLife S2 Medium Complete kit containing $25 \mu \mathrm{M}$ Xyl-SerC12, Xyl-Thr-C12, Gly-(Xyl)Ser-C12, or Gly-(Xyl)Thr-C12 for $48 \mathrm{~h}$.

Purification of Glycosylated Products. Purification of glycosylated products was carried out as previously reported, with some modifications. ${ }^{22}$ The glycosylated products were collected from the culture medium using a solid-phase extraction cartridge. To maximize the recovery of anionic products, tetrabutylammonium hydrogen sulfate $\left(\mathrm{TBA}-\mathrm{HSO}_{4}\right)$ was added into the culture medium as an ion pair reagent. First, $1 \mathrm{~mL}$ of $7.5 \mathrm{M}$ urea was added to the culture medium (about $1.5 \mathrm{~mL}$ ) and stirred well to dissolve the precipitate completely in the medium, followed by the addition of $2.5 \mathrm{~mL}$ of $20 \mathrm{mM}$ TBA- $\mathrm{HSO}_{4}$. Next, the solution was applied to the cartridge Strata-X, $60 \mathrm{mg}, 3 \mathrm{cc}$ (Phenomenex, CA) conditioned with methanol $(\mathrm{MeOH})$ and equilibrated with $10 \mathrm{mM}$ TBA-HSO ${ }_{4}$. After the cartridge was washed with $2 \mathrm{~mL}$ of water and $2 \mathrm{~mL}$ of $30 \% \mathrm{MeOH}$, the glycosylated products were eluted with $1 \mathrm{~mL}$ of $50 \% \mathrm{MeOH}, 70 \%$ of $\mathrm{MeOH}$, and $1 \mathrm{~mL}$ of $90 \% \mathrm{MeOH}$. The eluent fractions were collected, and the solvent was removed under reduced vacuum.

The samples extracted by SPE were passed through the cation exchange resin to remove the TBA ion, which can reduce MS sensitivity by its ion suppression effect. The residues were dissolved with $200 \mu \mathrm{L}$ of water/MeOH/2-propanol (1/1/1, v/ v) and passed through $200 \mu \mathrm{L}$ of the cation exchange resin (Muromachi Chemica, Japan) washed with $\mathrm{MeOH}$ and 

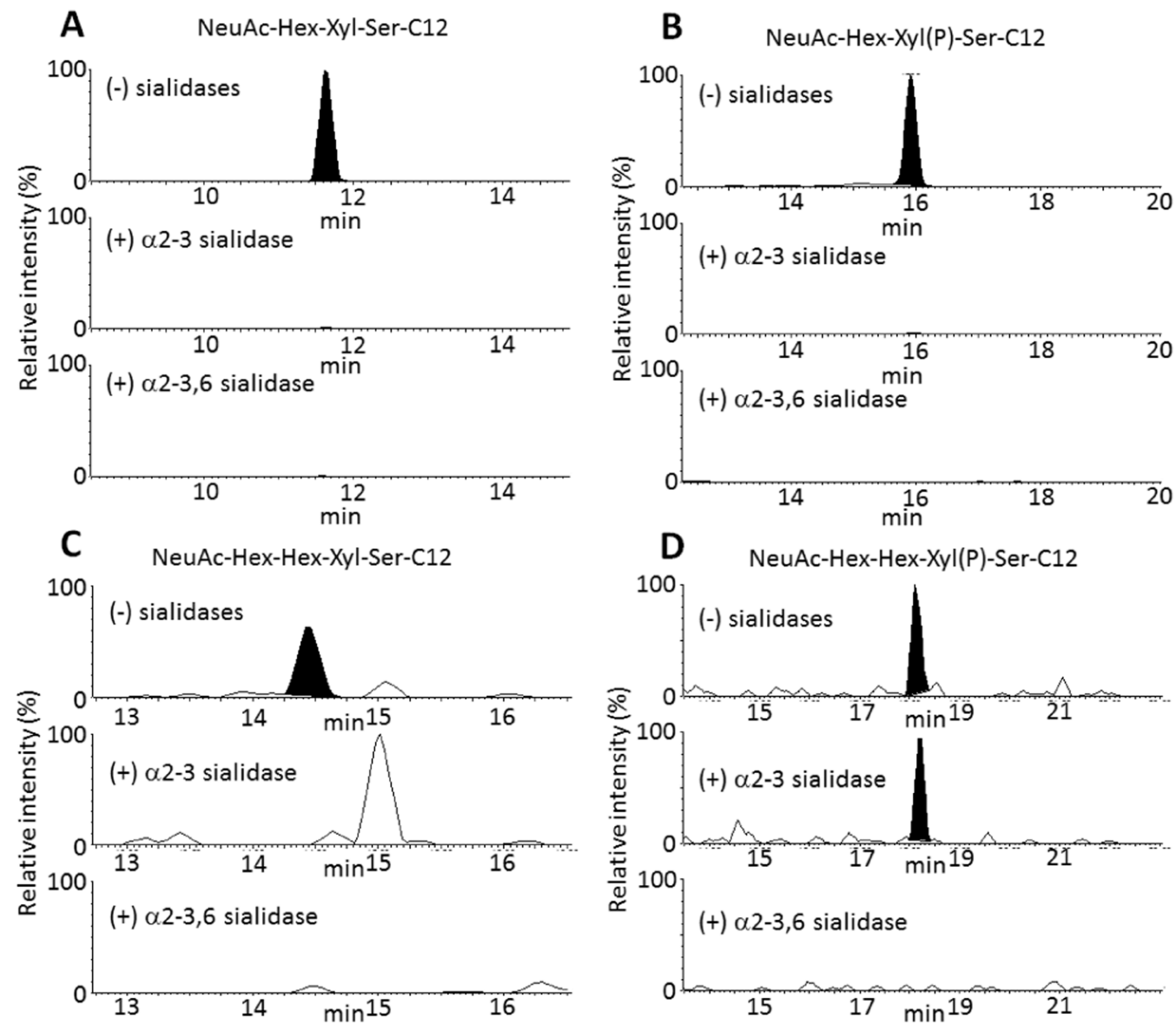

Figure 6. Comparison of EIC profiles before and after sialidase digestion. (A) EIC profiles of NeuAc-Hex-Xyl-Ser-C12; $m / z$ 870.4089. (B) EIC profiles of NeuAc-Hex-Xyl(P)-Ser-C12; $m / z$ 950.3752. (C) EIC profiles of NeuAc-Hex-Hex-Xyl-Ser-C12; $m / z$ 1032.4617. (D) EIC profiles of NeuAc-Hex-Hex-Xyl(P)-Ser-C12; $m / z$ 1112.4280. The ranges of the vertical axis are set equal.
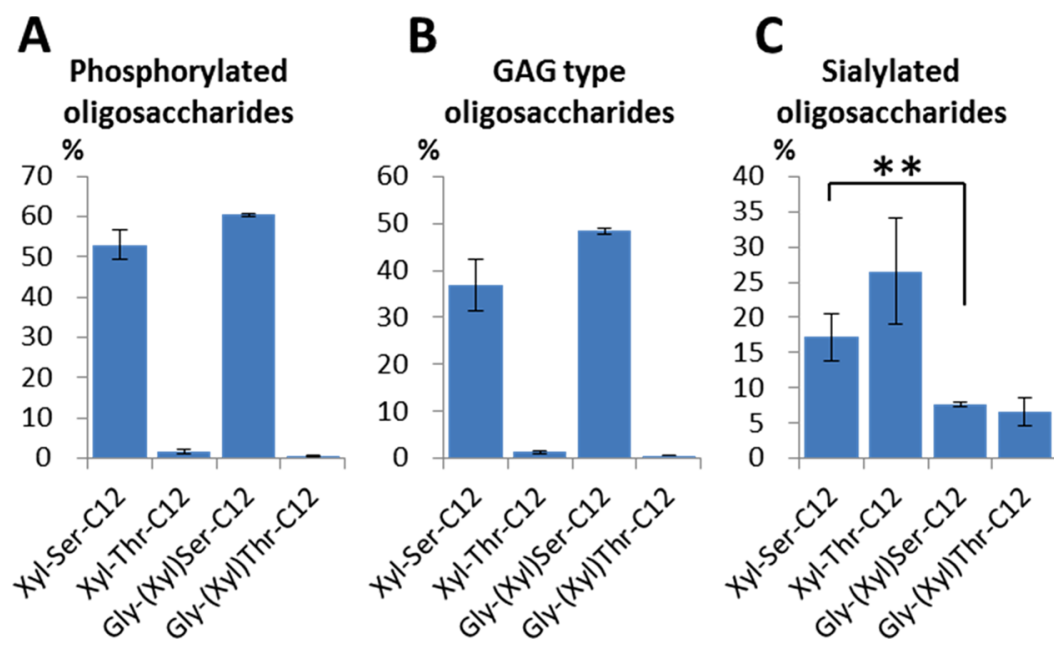

Figure 7. Total proportion of (A) phosphorylated, (B) GAG-type, and (C) sialyloligosaccharides elongated on the $\beta$-xylosides. Double asterisk (**) denotes $p<0.01$ in Student's $t$-test.

protonated with $2 \%$ formic acid. After removal of the solvent by vacuum distillation, the residues were stored at $-20{ }^{\circ} \mathrm{C}$.

Liquid Chromatography-Electrospray IonizationMass Spectrometry (LC-ESI-MS) of Glycosylated Products. All solvents and reagents used in the LC-MS experiment were of LC-MS grade. The glycosylated products and the digested samples were subjected to LC-ESI-MS analysis. MS analysis was performed by a Synapt G2-S (quadruple/time-of- flight mass spectrometer equipped with an ion mobility cell) coupled online by an ACQUITY UPLC H-Class Bio (Nihon Waters, Japan). Instrument control, data acquisition, and analysis were performed with MassLynx software v.4.1. The samples were dissolved in water/MeCN (1/3, v/v) and injected into ACQUITY UPLC BEH Glycan, $1.7 \mu \mathrm{m}(2.1 \mathrm{~mm}$ i.d. $\times$ $150 \mathrm{~mm}$; Nihon Waters, Japan). Fifty millimolar ammonium formate buffer ( $\mathrm{pH} 7.8$ ) was used as mobile phase $\mathrm{A}, \mathrm{MeCN}$ as 


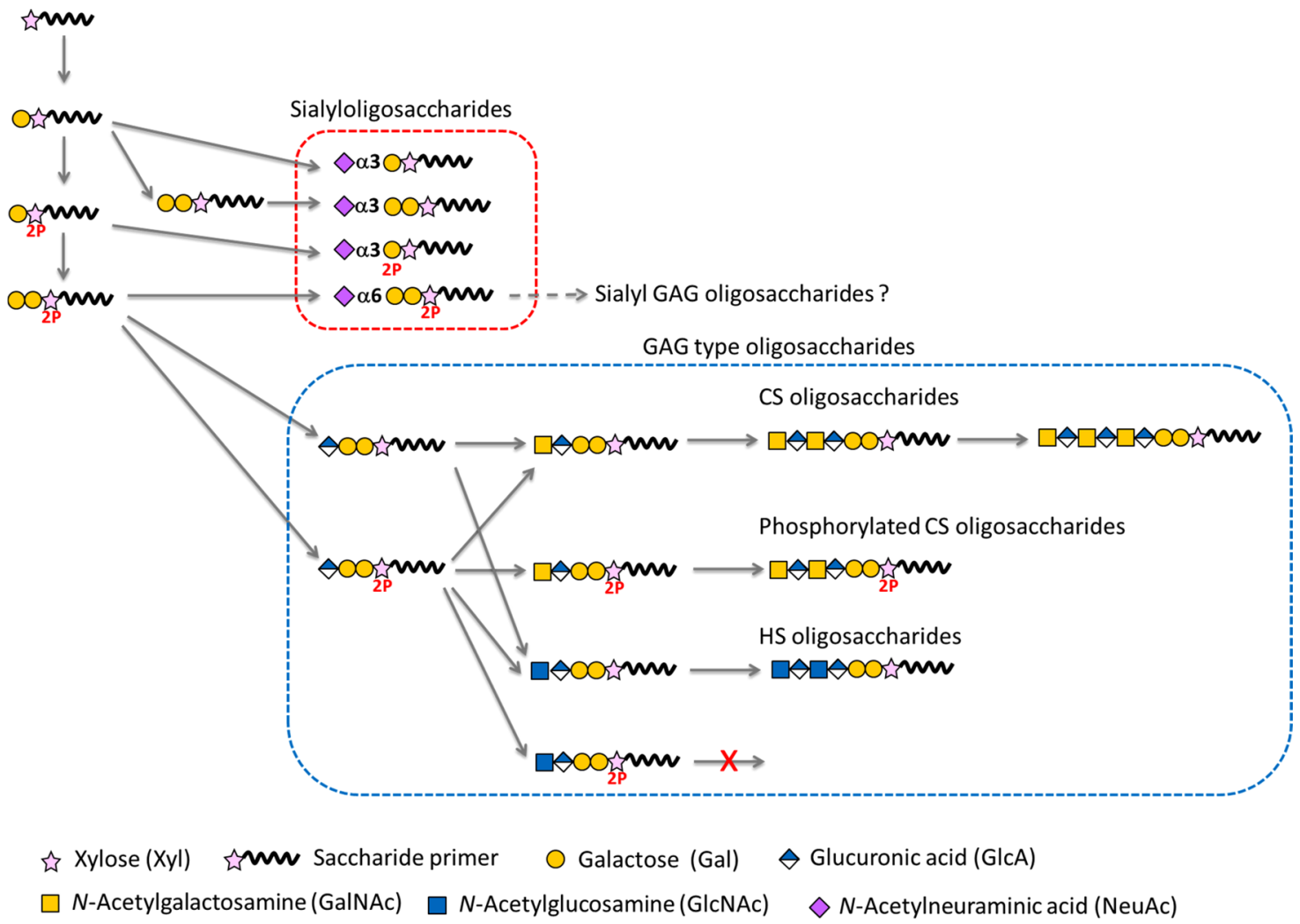

Figure 8. Speculated biosynthesis pathways of glycosylated products elongated on a saccharide primer. The biosynthesis is initiated with galactosylation on the Xyl. The disaccharide is phosphorylated for GAG-type oligosaccharide biosynthesis. The phosphorylated disaccharide is used as an intermediate of the GAG biosynthetic pathway. Dephosphorylation can occur during both GlcA addition on the phosphorylated trisaccharide and GalNAc addition on the phosphorylated tetrasaccharide. The phosphorylated trisaccharide can be modified by $\alpha 2-6$-linked NeuAc for sialyl GAG synthesis. Without the Xyl phosphorylation, the disaccharide can be capped by $\alpha 2$-3-linked NeuAc. Gal, HexA, and HexNAc residues can be sulfated during the biosynthesis.

mobile phase $\mathrm{B}$, and $20 \mathrm{mM}$ phosphoric acid in $50 \% \mathrm{MeCN}$ as mobile phase C. Before every analysis, the high-performance liquid chromatography system and the column were flushed with mobile phase $\mathrm{C}$ at $0.3 \mathrm{~mL} / \mathrm{min}$ for $3 \mathrm{~min}$ to avoid undesirable absorption of phosphorylated compounds and then washed with a mobile phase $\mathrm{A} /$ mobile phase $\mathrm{B}=50: 50$ solution at $0.3 \mathrm{~mL} / \mathrm{min}$ for $2 \mathrm{~min}$. The column was equilibrated with $95 \% \mathrm{~B}$, and the sample was eluted with a $95-50 \%$ B linear gradient for $40 \mathrm{~min}$ at a flow rate of $0.4 \mathrm{~mL} / \mathrm{min}$. The column temperature was set at $60{ }^{\circ} \mathrm{C}$. The $\mathrm{m} / z$ recorded ranged from 50 to 1500 in the resolution mode (about 20000 ). The MS was calibrated by $\mathrm{NaI}$, and the lock mass spray was set up with Leuenkephalin $\left(m / z\right.$ 554.2615: $\left.[\mathrm{M}-\mathrm{H}]^{-}\right)$. The quadruple profile was optimized to not detect the background phosphate ion. Other ESI conditions were set as follows: capillary voltage, -1.5 $\mathrm{kV}$; cone voltage, $30 \mathrm{~V}$; sample offset, $60 \mathrm{~V}$; source temperature, $120{ }^{\circ} \mathrm{C}$; desolvation temperature, $500{ }^{\circ} \mathrm{C}$; cone gas, $50 \mathrm{~L} / \mathrm{h}$; trap CE, 2; trans CE, 2.

All MS/MS spectra were recorded in a high definition multiple reaction monitoring mode. Wideband enhancement calibration was carried out using Glu-Fibrinopeptide B. Trap CE was set in accordance with the $m / z$ values of the precursor ions (Tables S5-S8). Assignment of the fragment ions was carried out following the nomenclature introduced by Domon and Costello. ${ }^{46}$

The proportions of glycosylated products were calculated by dividing the peak area of each product by the sum of peak areas of the glycosylated products.
Enzymatic Digestion of the Glycosylated Products. C$\mathrm{ABC}, \mathrm{C}-\mathrm{ACII}$, heparitinase I, heparitinase II, and heparitinase III were purchased from Seikagaku Corporation. $\beta$-DGalactosidase was purchased from Wako Pure Chemical Industries. $\alpha 2-3$ Sialidase was purchased from Takara Bio and $\alpha 2,3-6$ sialidase from Nakarai Tesque. The purified glycosylated products were dissolved in $100 \mu \mathrm{L}$ of water. For CS/DS digestion, $100 \mu \mathrm{L}$ of $100 \mathrm{mM}$ Tris $-\mathrm{HCl}$ buffer $(\mathrm{pH} 8.0)$ containing $250 \mathrm{mU}$ of $\mathrm{C}-\mathrm{ABC}, 25 \mathrm{mU}$ of C-ACII, and $0.005 \%$ BSA was added. For CS digestion, $100 \mathrm{mM}$ sodium acetate buffer ( $\mathrm{pH} 6.0$ ) containing $250 \mathrm{mU}$ of C-ACII and $0.005 \%$ BSA was added. For HS digestion, $100 \mathrm{mM}$ sodium acetate and 50 $\mathrm{mM}$ calcium acetate $(\mathrm{pH} 7.0)$ containing $250 \mathrm{mU}$ of heparitinase I, II, and III were added. For $\beta$-galactosidase digestion, $100 \mu \mathrm{L}$ of $100 \mathrm{mM}$ phosphate buffer ( $\mathrm{pH} 7.3$ ) containing $1000 \mathrm{U}$ of $\beta$-D-galactosidase was added. For $\alpha 2-3$ sialidase digestion, $100 \mathrm{mM}$ sodium acetate buffer $(\mathrm{pH} 5.5)$ and $10 \mu \mathrm{L}$ of a2-3 sialidase were added. For $\alpha 2,3-6$ sialidase digestion, $100 \mathrm{mM}$ sodium acetate buffer ( $\mathrm{pH}$ 5.0) containing $2000 \mathrm{mU}$ of $\alpha 2,3-6$ sialidase was added. The mixture solutions were maintained at $37{ }^{\circ} \mathrm{C}$ for $10 \mathrm{~min}(\alpha 2-3$ sialidase), $2 \mathrm{~h}$ (C$\mathrm{ABC}, \mathrm{ACII})$, or $17 \mathrm{~h}$ (HSases, $\beta$-galactosidase, $\alpha 2,3-6$ sialidase digestion). All of the reactions were quenched by heating for 30 $\mathrm{s}$ in a boiling water bath. The samples were passed through 200 $\mu \mathrm{L}$ of cation exchange resin, concentrated, and stored at -20 ${ }^{\circ} \mathrm{C}$ before use.

Statistical Analysis. All values are given as the mean \pm SD. Statistical analyses were performed using Student's $t$-test 
available in Microsoft Excel, and the level of significance is indicated in the figure when more than three independent experiments were performed.

\section{ASSOCIATED CONTENT}

\section{S Supporting Information}

The Supporting Information is available free of charge on the ACS Publications website at DOI: 10.1021/acsomega.7b00073.

Additional information includes NMR spectra of $\beta$ xylosides, results of enzymatic digestions, detected glycosylated products, MRM transitions, and experimental procedure for chemical synthesis (PDF)

\section{AUTHOR INFORMATION}

\section{Corresponding Author}

*E-mail: sato@bio.keio.ac.jp.

\section{ORCID}

Toshinori Sato: 0000-0002-4429-6101

\section{Notes}

The authors declare no competing financial interest.

\section{ACKNOWLEDGMENTS}

The authors deeply acknowledge Tetsuya Hirayama, Nobuo Kobayashi, and Hiroshi Fujita of the Seikagaku Corporation for their extensive support in chemical synthesis, GAG lyase digestion experiments, and critical reading.

\section{REFERENCES}

(1) Sasisekharan, R.; Raman, R.; Prabhakar, V. Glycomics approach to structure-function relationships of glycosaminoglycans. Annu. Rev. Biomed. Eng. 2006, 8, 181-231.

(2) Ly, M.; Laremore, T. N.; Linhardt, R. J. Proteoglycomics: recent progress and future challenges. OMICS 2010, 14, 389-399.

(3) Uyama, T.; Kitagawa, H.; Sugahara, K. Biosynthesis of Glycosaminoglycans and Proteoglycans. In Comprehensive Glycoscience; Elsevier, 2007; pp 79-104.

(4) Silbert, J. E.; Sugumaran, G. Biosynthesis of Chondroitin/ Dermatan Sulfate. IUBMB Life 2002, 54, 177-186.

(5) Wen, J.; Xiao, J.; Rahdar, M.; Choudhury, B. P.; Cui, J.; Taylor, G. S.; Esko, J. D.; Dixon, J. E. Xylose phosphorylation functions as a molecular switch to regulate proteoglycan biosynthesis. Proc. Natl. Acad. Sci. U.S.A. 2014, 111, 15723-15728.

(6) Izumikawa, T.; Sato, B.; Mikami, T.; Tamura, J.; Igarashi, M.; Kitagawa, $\mathrm{H}$. GlcUA $\beta 1-3 \mathrm{Gal} \beta 1-3 \mathrm{Gal} \beta 1-4 \mathrm{Xyl}(2-\mathrm{O}$-phosphate) Is the Preferred Substrate for Chondroitin N-Acetylgalactosaminyltransferase-1. J. Biol. Chem. 2015, 290, 5438-5448.

(7) Moses, J.; Oldberg, A.; Cheng, F.; Fransson, L.-A. Biosynthesis of the Proteoglycan Decorin Transient 2-Phosphorylation of Xylose during Formation of the Trisaccharide Linkage Region. Eur. J. Biochem. 1997, 248, 521-526.

(8) Okayama, M.; Kimata, K.; Suzuki, S. The Influence of pNitrophenyl $\beta$-D-Xyloside on the Synthesis of Proteochondroitin Sulfate by Slices of Embryonic Chick Cartilage. J. Biochem. 1973, 74, 1069-1073.

(9) Izumi, J.; Takagaki, K.; Nakamura, T.; Shibata, S.; Kojima, K.; Kato, I.; Endo, M. A Novel Oligosaccharide, Xylosyl $\beta 1-4$ Xylosyl $\beta 1$-(4Methylumbelliferone), Synthesized by Cultured Human Skin Fibroblasts in the Presence of 4-Methylumbelliferyl- $\beta$-D-Xyloside. J. Biochem. 1994, 116, 524-529.

(10) Lugemwa, F. N.; Esko, J. D. Estradiol- $\beta$-D-Xyloside, an Efficient Primer for Heparan Sulfate Biosynthesis. J. Biol. Chem. 1991, 266, 6674-6677.

(11) Abrahamsson, C.-O.; Ellervik, U.; Eriksson-Bajtner, J.; Jacobsson, M.; Mani, K. Xylosylated naphthoic acid-amino acid conjugates for investigation of glycosaminoglycan priming. Carbohydr. Res. 2008, 343, 1473-1477.

(12) Jacobsson, M.; Mani, K.; Ellervik, U. Effects of oxygen-sulfur substitution on glycosaminoglycan-priming naphthoxylosides. Bioorg. Med. Chem. 2007, 15, 5283-5299.

(13) Siegbahn, A.; Aili, U.; Ochocinska, A.; Olofsson, M.; Rönnols, J.; Mani, K.; Widmalm, G.; Ellervik, U. Synthesis, conformation and biology of naphthoxylosides. Bioorg. Med. Chem. 2011, 19, 4114-4126.

(14) Jacobsson, M.; Ellervik, U.; Belting, M.; Mani, K. Selective antiproliferative activity of hydroxynaphthyl-beta-D-xylosides. J. Med. Chem. 2006, 49, 1932-1938.

(15) Vassal-Stermann, E.; Duranton, A.; Black, A. F.; Azadiguian, G.; Demaude, J.; Lortat-Jacob, H.; Breton, L.; Vivès, R. R. A New CXyloside induces modifications of GAG expression, structure and functional properties. PLoS One 2012, 7, No. e47933.

(16) Pineau, N.; Carrino, D. A.; Caplan, A. I.; Breton, L. Biological evaluation of a new $\mathrm{C}$-xylopyranoside derivative (C-Xyloside) and its role in glycosaminoglycan biosynthesis. Eur. J. Dermatol. 2011, 21, 359-370.

(17) Kuberan, B.; Ethirajan, M.; Victor, X. V.; Tran, V.; Nguyen, K.; Do, A. "Click" xylosides initiate glycosaminoglycan biosynthesis in a mammalian cell line. ChemBioChem 2008, 9, 198-200.

(18) Victor, X. V.; Nguyen, T. K. N.; Ethirajan, M.; Tran, V. M.; Nguyen, K. V.; Kuberan, B. Investigating the elusive mechanism of glycosaminoglycan biosynthesis. J. Biol. Chem. 2009, 284, 2584225853.

(19) Tran, V. M.; Kuberan, B. Synthesis of fluorophore-tagged xylosides that prime glycosaminoglycan chains. Bioconjugate Chem. 2014, 25, 262-268.

(20) Kalita, M.; Quintero, M. V.; Raman, K.; Tran, V. M.; Kuberan, B. Synthesis and biomedical applications of xylosides. Methods Mol. Biol. 2015, 1229, 517-528.

(21) Freeze, H. H.; Etchison, J. R. A New Side of Xylosides and Their Close Relatives: Co-Localization Mapping of Glycosyl Transferases in the Functional Golgi. Trends Glycosci. Glycotechnol. 1996, 8, 65-77.

(22) Wang, Y.; Kumazawa, T.; Shiba, K.; Osumi, K.; Mizuno, M.; Sato, T. Glycosylation of $N \alpha$-lauryl- $O-(\beta$-D-xylopyranosyl)-L-serinamide as a saccharide primer in cells. Carbohydr. Res. 2012, 361, 33-40.

(23) Wang, L.-X.; Amin, M. N. Chemical and chemoenzymatic synthesis of glycoproteins for deciphering functions. Chem. Biol. 2014, 21, 51-66.

(24) van Rantwijk, F.; Woudenberg-van Oosterom, M. W.; Sheldon, R. A. Glycosidase-catalysed synthesis of alkyl glycosides. J. Mol. Catal. B: Enzym. 1999, 6, 511-532.

(25) Johansson, E.; Hedbys, L.; Larsson, P. Enzymatic synthesis of monosaccharide-amino acid conjugates Enzyme Microb. Technol. 1991, 13, 781787 10.1016/0141-0229(91)90060-N.

(26) Eneyskaya, E. V.; Brumer, H.; Backinowsky, L. V.; Ivanen, D. R.; Kulminskaya, A. A.; Shabalin, K. A.; Neustroev, K. N. Enzymatic synthesis of beta-xylanase substrates: transglycosylation reactions of the beta-xylosidase from Aspergillus sp. Carbohydr. Res. 2003, 338, $313-325$

(27) Muzard, M.; Aubry, N.; Plantier-Royon, R.; O’Donohue, M.; Rémond, C. Evaluation of the transglycosylation activities of a GH 39 $\beta$-D-xylosidase for the synthesis of xylose-based glycosides. J. Mol. Catal. B: Enzym. 2009, 58, 1-5.

(28) Kadi, N.; Crouzet, J. Transglycosylation reaction of endoxylanase from Trichoderma longibrachiatum. Food Chem. 2008, 106, 466-474.

(29) Ajisaka, K.; Miyasato, M.; Ishii-Karakasa-Ikuko. Synthesis fo OLinked glycopeptide by Endo-GalNAc-ase S Assisted Transglycosylation. Biosci. Biotechnol. Biochem. 2001, 1240-1243.

(30) Eneyskaya, E. V.; Iii, B.; Backinowsky, L. V.; Ivanen, D. R.; Kulminskaya, A. A.; Shabalin, K. A.; Neustroev, N. Enzymatic synthesis of b-xylanase substrates: transglycosylation reactions of the bxylosidase from Aspergillus sp. Carbohydr. Res. 2003, 338, 313-325.

(31) Kurakake, M.; Ito, Y.; Komaki, T. Synthesis of sugar fatty acid esters using a $\beta$-xylosidase from Aspergillus awamori for transxylosylation. Biotechnol. Lett. 2011, 33, 2453-2456. 
(32) Nilsson, K. G. I.; Scigelova, M. Synthesis of Glycosylated Serine and Threonine Derivatives Employing Glycosidases. Biotechnol. Lett. 1994, 16, 671-676.

(33) Groll, M.; Götz, M.; Kaiser, M.; Weyher, E.; Moroder, L. TMC95-based inhibitor design provides evidence for the catalytic versatility of the proteasome. Chem. Biol. 2006, 13, 607-614.

(34) Delbaere, L.; Higham, M.; Kamenar, B.; Kent, P.; Prout, C. The crystal and molecular structure of $O$-( $\beta$-D-xylopyranosyl)-L-serine and its copper (II) complex and some of their reactions. Biochim. Biophys. Acta, Gen. Subj. 1972, 286, 441-444.

(35) Ekborg, G.; Klinger, M.; Rodën, L.; Wjesen, J.; Schutzbach, J. S.; Huang, D. H.; Krishna, N. R.; Anantharamaiah, G. M. Synthesis of 3$O-\beta$-D-xylopyranosyl-L-serine (xylosylserine) and $O-\beta$-D-galactopyranosyl-(1-4)-O- $\beta$-D-xylopyranosyl-L-serine (galactosylxylosylserine) and use of the synthetic products for detection of galactosyltransferase I activity in rat liver. Glycoconjugate J. 1987, 4, 255-266.

(36) Greve, H.; Kresse, H. Secretion of unphosphorylated and phosphorylated xyloside-induced glycosaminoglycan chains. Glycoconjugate J. 1988, 5, 175-183.

(37) Moses, J.; Oldberg, A.; Fransson, L.-A. Initiation of galactosaminoglycan biosynthesis. Separate galactosylation and dephosphorylation pathways for phosphoxylosylated decorin protein and exogenous xyloside. Eur. J. Biochem. 1999, 260, 879-884.

(38) Prydz, K.; Dalen, K. T. Synthesis and sorting of proteoglycans. J. Cell Sci. 2000, 205, 193-205.

(39) Sugahara, K.; Tsuda, H.; Yoshida, K.; Yamada, S.; de Beer, T.; Vliegenthart, J. F. G. Structure Determination of the Octa- and Decasaccharide Sequences Isolated from the Carbohydrate-Protein Linkage Region of Porcine Intestinal Heparin. J. Biol. Chem. 1995, 270, 22914-22923.

(40) Cheng, F.; Heinegârd, D.; Fransson, L.; Bayliss, M.; Bielicki, J.; Hopwood, J.; Yoshida, K. Variations in the chondroitin sulfate-protein linkage region of aggrecans from bovine nasal and human articular cartilages. J. Biol. Chem. 1996, 271, 28572-28580.

(41) Tuytten, R.; Lemière, F.; Witters, E.; Van Dongen, W.; Slegers, H.; Newton, R. P.; Van Onckelen, H.; Esmans, E. L. Stainless steel electrospray probe: a dead end for phosphorylated organic compounds? J. Chromatogr. A 2006, 1104, 209-221.

(42) Antonopoulos, A.; Favetta, P.; Jacquinet, J.-C.; Lafosse, M. Tandem mass spectrometry for the characterisation of sulphatedphosphorylated analogues of the carbohydrate-protein linkage region of proteoglycans. J. Mass Spectrom. 2005, 40, 1628-1636.

(43) Kenny, D. T.; Issa, S. M. A.; Karlsson, N. G. Sulfate migration in oligosaccharides induced by negative ion mode ion trap collisioninduced dissociation. Rapid Commun. Mass Spectrom. 2011, 25, 26112618.

(44) Gomez Toledo, A.; Nilsson, J.; Noborn, F.; Sihlbom, C.; Larson, G. Positive Mode LC-MS/MS Analysis of Chondroitin Sulfate Modified Glycopeptides Derived from Light and Heavy Chains of The Human Inter- $\alpha$-Trypsin Inhibitor Complex. Mol. Cell. Proteomics 2015, 14, 3118-3131.

(45) Wang, Y.; Yang, X.; Yamagata, S.; Yamagata, T.; Sato, T. Involvement of Ext1 and heparanase in migration of mouse FBJ osteosarcoma cells. Mol. Cell. Biochem. 2013, 373, 63-72.

(46) Domon, B.; Costello, C. E. A systematic nomenclature for carbohydrate fragmentations in FAB-MS/MS spectra of glycoconjugates. Glycoconjugate J. 1988, 5, 397-409. 